\title{
Amplitude calculation near a period-doubling bifurcation: An example
}

\author{
Wiesenfeld, K.; Pedersen, Niels Falsig
}

Published in:

Physical Review A

Link to article, DOI:

10.1103/PhysRevA.36.1440

Publication date:

1987

Document Version

Publisher's PDF, also known as Version of record

Link back to DTU Orbit

Citation (APA):

Wiesenfeld, K., \& Pedersen, N. F. (1987). Amplitude calculation near a period-doubling bifurcation: An example. Physical Review A, 36(3), 1440-1444. https://doi.org/10.1103/PhysRevA.36.1440

\section{General rights}

Copyright and moral rights for the publications made accessible in the public portal are retained by the authors and/or other copyright owners and it is a condition of accessing publications that users recognise and abide by the legal requirements associated with these rights.

- Users may download and print one copy of any publication from the public portal for the purpose of private study or research.

- You may not further distribute the material or use it for any profit-making activity or commercial gain

- You may freely distribute the URL identifying the publication in the public portal

If you believe that this document breaches copyright please contact us providing details, and we will remove access to the work immediately and investigate your claim. 


\title{
Amplitude calculation near a period-doubling bifurcation: An example
}

\author{
K. Wiesenfeld \\ Brookhaven National Laboratory, Upton, New York 11973-5000 \\ N. F. Pedersen \\ Physics Laboratory I, The Technical University of Denmark, DK-2800 Lyngby, Denmark
}

(Received 26 March 1987)

\begin{abstract}
For the rf-driven Josephson junction, the dynamical behavior is studied near a period-doubling transition. The center-manifold theorem simplifies the problem and enables us to study only a firstorder system, the parameters of which are expressed in terms of the Josephson-junction parameters.
\end{abstract}

\section{INTRODUCTION}

Recently there has been a lot of theoretical as well as experimental interest in the behavior of physical systems near a period-doubling instability. In this paper we discuss explicitly the Josephson junction, which is a wellestablished nonlinear system accessible to experimental investigations.

There are three motives for the present study: The first two are pedagogical, the third practical.

(i) Theoretically, period-doubling bifurcations are well understood, proceeding from a bifurcation analysis of a discrete Poincaré map for the dynamics. As a practical matter, however, there is a gap in this understanding because the return map typically must be determined numerically from the underlying (differential) equations of motion. Thus one usually asserts that there is "some transformation" (whose explicit form is expected to be quite complicated) connecting the experimentally accessible control parameters with the theoretically convenient bifurcation parameters. The present work provides a rare example where this transformation is written down explicitly. Our method uses an "indirect" technique that is rather simple, and may be usefully applied to other problems.

(ii) An insufficiently appreciated fact is that there are two kinds of period-doubling bifurcation, called supercritical and subcritical, respectively. The supercritical type is by far the more familiar since it can be directly observed in experiments and numerical simulations; in contrast, a subcritical period doubling must be inferred from indirect evidence. Besides the usual parameter that sweeps through the period-doubling bifurcation point, the example that we study has a second parameter that continuously tunes the system between these two types (i.e., we study a codimension-2 bifurcation).

(iii) Josephson-junction systems continue to play an important role in the study of nonlinear dynamics. For example very recent work concerning the effects of swept parameters ${ }^{1,2}$ and periodic and random perturbations ${ }^{3-9}$ in the vicinity of period-doubling bifurcations are well suited to testing by Josephson-junction circuits. In this paper we consider a parameter regime directly accessible by real experiments, so establishing the explicit connection between experimentally accessible and theoretically convenient parameters is of some practical significance.

The paper is organized in the following way. Section II contains a qualitative description of the bifurcation. Section III gives analytical calculations for the Josephson junction. In Sec. IV the two approaches are compared and the bifurcation parameters are derived explicitly.

\section{QUALITATIVE DESCRIPTION OF THE BIFURCATION}

Near the onset of a dynamical instability, it is often possible to drastically reduce the complexity of the governing equations. That is, for all practical purposes it is sufficient to consider a very simple equation rather than the full set of ordinary differential equations (ODE's). In this paper we focus on the case of period doubling; then the essential dynamics is captured by a first-order nonlinear differential equation, regardless of the complexity of the original equations.

The origin of this simplification lies in the centermanifold theorem from the geometric theory of differential equations. ${ }^{10-12}$ The situation is illustrated in Fig. 1, which depicts a stable periodic orbit $\mathbf{X}$ in an $N$ dimensional phase space with a Poincaré section $P$ taken transverse to $\mathbf{X}$. Typically, any nearby orbit will successively intersect $P$ in a haphazard way as it relaxes toward $\mathbf{X}$, and the dynamics is described by an $(N-1)-$ dimensional iterative map. The situation is quite different near a bifurcation point: Near a period doubling successive iterates quickly relax to a one-dimensional curve (the center manifold) and only slowly evolve along that curve. It is the slow dynamics that captures the essential behavior, and this depends on only a single coordinate, say $x$. (The crucial role of the slow variables has been emphasized by Haken ${ }^{13}$ in what he picturesquely calls the "slaving principle;" the center-manifold theorem gives the formal justification for this cornerstone of the adiabatic elimination method.)

The dynamics on the center manifold is given by an iterative map

$$
x_{n+1}=f\left(x_{n} ; \lambda\right)
$$

which in principle can be derived from the starting 

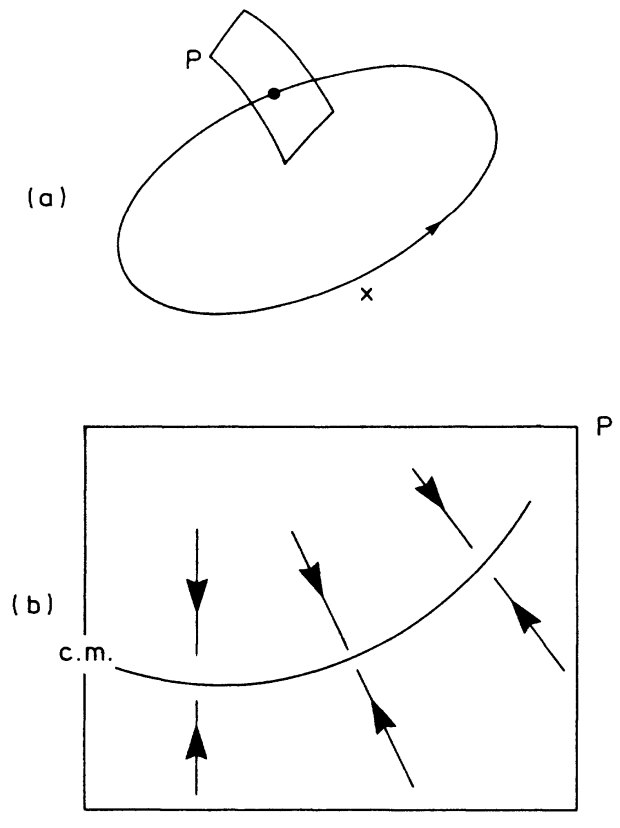

FIG. 1. (a) Periodic orbit $\mathbf{X}$ forms a closed trajectory in phase space, and is cut by a transverse section $P$. (b) Nearby trajectories quickly relax toward a one-dimensional center manifold (c.m.) near the onset of a period-doubling bifurcation. On the c.m., the dynamics evolves relatively slowly.

differential equations which depend on some set of parameters $\lambda$. In practice, however, this is an extremely difficult task and so Eq. (2.1) is taken as a starting point of a dynamical systems analysis.

Choosing $x=0$ to coincide with the point $X \cap P$ [which is a fixed point of Eq. (2.1)], we expand $f\left(x_{n} ; \lambda\right)$ in a Taylor series

$$
x_{n+1}=\alpha x_{n}+\beta x_{n}^{2}+\gamma x_{n}^{3}+\cdots .
$$

Near a period doubling, $\alpha=-1-\mu / 2$, where $\mu$ is a small parameter ( $\mu=0$ at the bifurcation point). Looking at the second iterate yields, up to third order, ${ }^{12}$

$$
x_{n+2}-x_{n}=\mu x_{n}-a x_{n}^{3}+\cdots,
$$

where $a=2\left(\beta^{2}+\gamma\right)$. There are two distinct kinds of period-doubling bifurcations, depending on the sign of $a$. If $a>0$, we have the more familiar supercritical bifurcation: As depicted in Fig. 2(a), a stable period-1 orbit goes unstable at the bifurcation point giving birth to a stable period-2 orbit. If $a<0$ instead, the period doubling is subcritical: At the bifurcation point a stable period-1 annihilates an unstable period- 2 orbit, leaving behind a single unstable period-1 solution [Fig. 2(b)]. In the latter case, higher-order terms must be included to determine what attractors the system might evolve toward; for the problem considered below, the quintic term is sufficient

$$
x_{n+2}-x_{n}=\mu x_{n}-a x_{n}^{3}-x_{n}^{5}+\cdots .
$$

The absence of the quartic term can be explained as fol-

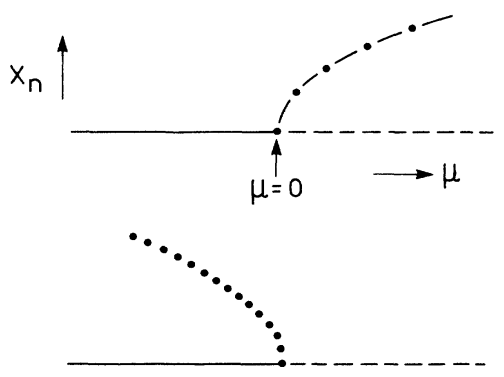

(a)



(c)

FIG. 2. Bifurcation diagrams for period doubling in various cases: (a) supercritical, (b) subcritical, (c) subcritical with stabilizing quintic term included. - , stable period $1 ;-\ldots-$, unstable period $1 ; \cdot \cdot$, stable period $2 ;. .$. , unstable period 2 . The final case displays hysteresis as the control parameter $\mu$ is swept back and forth across the period-doubling bifurcation point $\mu=0$.

lows. The proper scaling is given by the condition that all terms retained on the right-hand side are of the same order, so

$$
\mu \sim O\left(x^{4}\right), \quad a \sim O\left(x^{2}\right) .
$$

This scaling leads to the vanishing of the quartic term in Eq. (2.4). The coefficient of the quintic term has been taken to be negative; the sign is crucial to stabilize the dynamics, while the magnitude can always be made unity by a simple linear scaling. The quintic term leads to Fig. 2(c) in the subcritical $(a<0)$ case; from an experimental point of view, this case is distinguished from the supercritical case due to the presence of hysteresis as indicated by the arrows in Fig. 2(c), and the associated "sudden appearance" of a finite half-harmonic Fourier amplitude rather than the more familiar square-root law found at the onset of the supercritical period-doubling bifurcation.

To make an explicit connection between the original ODE and Eq. (2.4) requires expressions for the parameters $\mu$ and $a$ in terms of the original parameters $\lambda$. In principle, there is a well-defined procedure for doing this by directly calculating the equation for the center manifold, but in practice this is a forbidding task. ${ }^{14}$ (The situation is somewhat easier ${ }^{12}$ in the case of bifurcations of fixed points rather than the present case of periodic orbits.)

The point of this paper is to demonstrate explicitly the connection between the driven Josephson-junction equation and the normal form Eq. (2.4). Our strategy bypasses any direct center-manifold reduction; rather, we take a two-step approach. By using harmonic balance on the ODE, we derive an expression for the half-harmonic 
Fourier amplitude. This quantity is proportional to $x_{n}$ at the fixed point of Eq. (2.4), and we use this fact to derive expressions for $\mu$ and $a$ in terms of the parameters appearing in the ODE.

\section{THE JOSEPHSON CIRCUIT CALCULATION}

We begin with the usual equation governing the dynamics of the driven Josephson junction ${ }^{15}$ (Fig. 3)

$$
I_{\mathrm{dc}}+I_{\mathrm{rf}} \cos (\omega t)=C \dot{V}+V / R+I_{0} \sin \phi,
$$

where $I_{\mathrm{dc}}$ is the dc bias current, $I_{\mathrm{rf}}$ is the current at frequency $\omega, I_{0}$ is the maximum supercurrent, $R$ is the resistance, $C$ the capacitance, and $\phi$ and $V$ are the phase difference and voltage across the junction, respectively. Introducing the Josephson relation

$$
V=(\hbar / 2 e) \dot{\phi},
$$

where $\hbar$ is Planck's constant divided by $2 \pi$ and $-e$ the electron charge, Eq. (3.1) may be rewritten as

$$
\ddot{\phi}+\dot{\phi} / \tau+\omega_{0}^{2} \sin \phi=\omega_{0}^{2}\left[\rho_{0}+\rho_{1} \cos (\omega t)\right],
$$

where $\tau=R C, \omega_{0}^{2}=2 e I_{0} / \hbar C, \rho_{0}=I_{\mathrm{dc}} / I_{0}$, and $\rho_{1}=I_{\mathrm{rf}} / I_{0}$.

In the following the junction is assumed to be dc biased in the zero-voltage mode $\left(I_{\mathrm{dc}}<I_{0}\right)$. For small rf levels $\left(\rho_{1} \ll 1\right)$ a linearization of Eq. (3.3) gives a solution for $\phi$ which is

$$
\phi=\phi_{0}+\phi_{1} \cos (\omega t-\theta)
$$

where

$$
\begin{aligned}
& \sin \phi_{0}=I_{\mathrm{dc}} / I_{0}, \\
& \phi_{1}=\omega_{0}^{2} \rho_{1}\left[\left(\omega_{p}^{2}-\omega^{2}\right)^{2}+(\omega / \tau)^{2}\right]^{-1 / 2},
\end{aligned}
$$

and

$$
\tan \theta=(\omega / \tau)\left(\omega_{p}^{2}-\omega^{2}\right)^{-1}
$$

Here the plasma frequency $\omega_{p}$ is given by

$$
\omega_{p}^{2}=\omega_{0}^{2} \cos \phi_{0} \text {. }
$$

In order to study period-doubling bifurcations we put

$$
\omega \approx 2 \omega_{p},
$$

since this leads to a bifurcation at relatively low values of $\rho_{1}$. In Ref. 16 it was shown that if the frequency condition Eq. (3.9) is precisely satisfied, half-harmonic generation occurs if the threshold condition

$$
\phi_{1} \tan \phi_{0}>2 / Q
$$

is satisfied. Here $Q=\omega_{p} \tau$.

In order to compute period-doubled solutions we insert

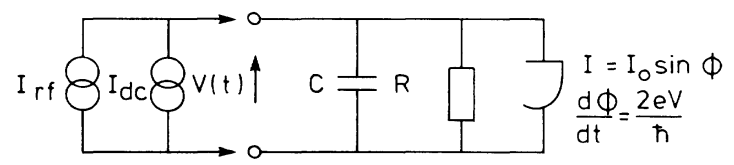

FIG. 3. Model circuit for the Josephson junction.

$$
\begin{aligned}
\phi= & \phi_{0}+\phi_{1} \cos (\omega t-\theta)+y \cos (\omega t / 2) \\
& +x \sin (\omega t / 2)
\end{aligned}
$$

into Eq. (3.3), expanding $\sin \phi$ in terms of the small quantities $\phi_{1}, x$, and $y$. The order of the expansion which is necessary may be judged as follows. An expansion to first order gives the small signal plasma resonance, Eqs. (3.6) and (3.7). An expansion to second order gives a subharmonic voltage component growing exponentially in time $e^{16}$ when the threshold condition Eq. (3.10) is satisfied. An expansion to third order in $\phi_{1}, x$, and $y$ will give a saturation of the half-harmonic voltage component, and hence determines its amplitude to lowest approximation.

Inserting Eq. (3.9) into Eq. (3.7) we find

$$
\tan \theta=-\left(\omega / \omega_{p}\right) / 3 Q,
$$

so that for $Q \gg 1$ we may take $\theta=\pi$ in Eq. (3.11). Balancing terms proportional to $\cos (\omega t / 2)$ and $\sin (\omega t / 2)$, respectively, yields after some algebraic manipulations the following two equations: ${ }^{17}$

$$
\begin{aligned}
0= & \frac{1}{4} y \omega^{2}+x \omega / 2 \tau+\frac{1}{2} y \phi_{1} \omega_{0}^{2} \sin \phi_{0}+y \omega_{0}^{2} \cos \phi_{0} \\
& -\frac{1}{4} \omega_{0}^{2} \cos \phi_{0}\left[\phi_{1}^{2} y+\frac{1}{2}\left(y^{3}+x^{2} y\right)\right], \\
0= & -\frac{1}{4} x \omega^{2}-y \omega / 2 \tau-\frac{1}{2} x \phi_{1} \omega_{0}^{2} \sin \phi_{0}+x \omega_{0}^{2} \cos \phi_{0} \\
& -\frac{1}{4} \omega_{0}^{2} \cos \phi_{0}\left[\phi_{1}^{2} x+\left(x^{3}+y^{2}\right) / 2\right] .
\end{aligned}
$$

The amplitudes $x$ and $y$ can be determined from Eqs. (3.13) and (3.14). The trivial solutions $x=y=0$ corresponds to the always present period-1 solution. A nontrivial solution is most conveniently found by introducing

$$
x=r \cos \beta, \quad y=r \sin \beta .
$$

Inserting Eq. (3.15) into Eqs. (3.13) and (3.14) and eliminating $\beta$, we find after some calculations that the amplitude $r$ of the subharmonic oscillation is given by ${ }^{17}$

$$
\begin{aligned}
r^{2}= & \pm(8 / Q)\left\{\left[\phi_{1} \tan \phi_{0} /(2 / Q)\right]^{2}-\left(\omega / 2 \omega_{p}\right)^{2}\right\}^{1 / 2} \\
& +8\left[1-\left(\omega / 2 \omega_{p}\right)^{2}\right] .
\end{aligned}
$$

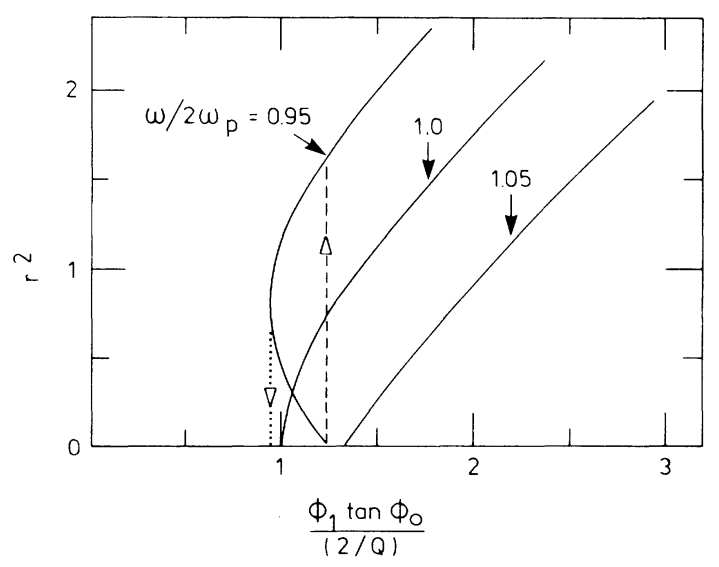

FIG. 4. Square of the half-harmonic amplitude $r$ as a function of the parameter $\phi_{1} \tan \phi_{0} /(2 / Q)$. Calculated from Eq. (3.1) with $Q=8$. 
This is the desired result, giving the amplitude of the half-harmonic Fourier amplitude in terms of the parameters of the governing ODE Eq. (3.3). Figure 4 shows $r^{2}$ plotted as a function of $\phi_{1} \tan \phi_{0} /(2 / Q)$ with $\left(\omega / 2 \omega_{p}\right)$ as a parameter, as calculated from Eq. (3.16). For $\omega=2 \omega_{p}$ we see that the amplitude of subharmonic oscillations becomes nonzero when $\phi_{1} \tan \phi_{0} /(2 / Q)=1$, in agreement with Eq. (3.10). This condition separates the supercritical period-doubling case from the subcritical case, as can be seen by comparing Figs. 2 and 4.

\section{COMPARISON WITH NORMAL FORM}

Armed with an expression for $r$, we return to the reduced dynamical picture. In the vicinity of a perioddoubling bifurcation, the center-manifold dynamics obeys

$$
x_{n+2}-x_{n}=\mu x_{n}-a x_{n}^{3}-x_{n}^{5}+O\left(x_{n}^{7}\right) .
$$

Recall that $x_{n}$ measures the displacement along the center manifold, where $x_{n}=0$ corresponds to the place where the period-1 orbit intersects the Poincare section. More importantly for our purposes, $x_{n}$ is proportional to the halfharmonic Fourier amplitude $r$. Equation (4.1) encompasses both the supercritical case [ $a>0$, Fig. 2(a)] and the subcritical case [ $a<0$, Fig. $2(\mathrm{c})]$.

We are interested in period-2 solutions of Eq. (4.1), obtained by setting $x_{n+2}$ equal to $x_{n}$. There immediately follows

$$
x_{n}^{2}=-a / 2 \pm\left[(a / 2)^{2}+\mu\right]^{1 / 2} .
$$

This should be compared with Eq. (3.16). These expressions are identical if we make the identifications

$x_{n}^{2}=r^{2} / 8$,

$a=-2\left[1-\left(\omega / 2 \omega_{p}\right)^{2}\right]$,

$\mu=\frac{1}{Q^{2}}\left[\left(\frac{\phi_{1} \tan \phi_{0}}{(2 / Q)}\right]^{2}-\left[\frac{\omega}{2 \omega_{p}}\right]^{2}\right]-\left[1-\left(\frac{\omega}{2 \omega_{p}}\right]^{2}\right]^{2}$.

Equations (4.3) and (4.4) are the main results of this paper, giving explicit expressions for the center-manifold parameters $a$ and $\mu$ in terms of the original control parameters of the ODE Eq. (3.3).

We close with three remarks. First, although our calculations only made use of "static" solutions of the iterative mapping, the validity of the map (and the specific identification of the parameters) is not restricted to these special solutions. In fact, this equation should give good results for the relaxation to the asymptotic states, the effects of external noise, ${ }^{3,4,9,18}$ and also for phenomena associated with quasistatic variation of parameters,, , Moreover, a straightforward extension of the calculation presented here will recover the relevant equation for near-resonantly perturbed period-doubling bifurcations ${ }^{5}$ which have now been seen in a variety of experiments, $5,14,19-22$ including those on the Josephson-junction circuit. $^{6}$

Second, recall that the sign of $a$ determines whether the bifurcation is supercritical or subcritical. Thus, we see from Eq. (4.3) that if $\left(\omega / 2 \omega_{p}\right)>1$ the bifurcation is supercritical, and if $\left(\omega / 2 \omega_{p}\right)<1$ it is subcritical. [The crossover point $\left(\omega / 2 \omega_{p}\right)=1$ determines a codimension- 2 bifurcation.] This behavior was already seen in Fig. 4; it is gratifying - but by no means to be expected generallythat the simple condition from the reduced equation remains a simple condition in terms of the original control parameters.

Finally, although Eq. (4.4) is a complicated functionin fact, this expression does not really do justice to the complexity, since it uses the quantities $\phi_{0}$ and $\phi_{1}$, which must in turn be evaluated via Eq. (3.6) and the transcendental Eq. (3.5) — the expected behavior as a single control parameter is varied is qualitatively the same as if $\mu$ itself is varied. The reason is that (barring exceptional choices of parameters) a small change from criticality of any parameter, say $\omega_{0}$, amounts to a similarly small change in $\mu$, i.e.,

$$
\mu=F\left(\tau, \omega_{0}, \rho_{0}, \rho_{1}\right)
$$

implies that

$$
\Delta \mu=\left(\delta F / \delta \omega_{0}\right) \Delta \omega_{0},
$$

where the partial derivative is evaluated at the bifurcation point. Thus, any bifurcation diagram is likely to look just like the ones generated in Fig. 2; this has already been demonstrated in Fig. 4.

\section{ACKNOWLEDGMENTS}

We thank A. Davidson, G. Eriksen, J. Bindslev Hansen, and H. Svensmark for stimulating discussions. The work at Brookhaven National Laboratory was supported by the Division of Material Sciences, U.S. Department of Energy, under Contract No. DE-AC02-76CH00016.
${ }^{1}$ R. Kapral and P. Mandel, Phys. Rev. A 32, 1076 (1985).

${ }^{2}$ B. Morris and F. Moss, Phys. Lett. A 118, 117 (1976).

${ }^{3}$ J. Heldstab, H. Thomas, T. Geisel, and G. Radons, Z. Phys. B 50, 141 (1983).

${ }^{4}$ K. Wiesenfeld, J. Stat. Phys. 38, 1071 (1985).

${ }^{5}$ P. Bryant and K. Wiesenfeld, Phys. Rev. A 33, 2525 (1986).

${ }^{6}$ H. Svensmark, J. Bindslev Hansen, and N. F. Pederson, Phys. Rev. A 35, 1457 (1987).

${ }^{7}$ K. Wiesenfeld and B. McNamara, Phys. Rev. Lett. 55, 13 (1985); Phys. Rev. A 33, 629 (1986).

${ }^{8}$ D. Hackenbracht and K.-H. Hock, J. Phys. C 19, 4095 (1986).
${ }^{9}$ J. Crutchfield and B. A. Huberman, Phys. Lett. 77A, 407 (1980).

${ }^{10}$ J. E. Marsden and M. McCraken, The Hopf Bifurcation and Its Application (Springer-Verlag, New York, 1976).

${ }^{11} \mathrm{~J}$. Carr, Applications of the Center Manifold Theory (SpringerVerlag, New York, 1981).

12J. Guckenheimer and P. Holmes, Nonlinear Oscillations, Dynamical Systems and Bifurcations of Vector Fields (Springer-Verlag, New York, 1983).

${ }^{13}$ See, e.g., H. Haken, Synergetics: An Introduction (SpringerVerlag, New York, 1977). 
${ }^{14}$ See, however, K. Wiesenfeld and N. B. Tufillaro, Physica D (to be published).

${ }^{15}$ A. Barone and G. Paterno, Physics and Applications of the Josephson Effect (Wiley-Interscience, New York, 1982).

${ }^{16}$ N. F. Pederson, M. R. Samuelsen, and K. Saermark, J. Appl. Phys. 44, 5120 (1973).

${ }^{17}$ N. F. Pedersen (unpublished).
${ }^{18}$ C. Jeffries and K. Wiesenfeld, Phys. Rev. A 31, 1077 (1985).

${ }^{19}$ B. Derighetti, M. Ravani, R. Stoop, P. F. Meier, E. Brun, and R. Badii, Phys. Rev. Lett. 55, 1746 (1985).

${ }^{20}$ S. Martin and W. Martienssen, Phys. Rev. A 34, 4523 (1986).

${ }^{21}$ P. Pieranski and J. Malecki, Phys. Rev. A 34, 582 (1986).

${ }^{22} \mathrm{H}$. Savage and C. Adler (private communication). 